\title{
Introduction to the RE'12 special issue
}

\author{
Pete Sawyer
}

Published online: 29 May 2013

(C) Springer-Verlag London 2013

\section{Editor's introduction}

This edition of the Requirements Engineering Journal is devoted to extended versions of the best papers from the 20th IEEE International Conference on Requirements Engineering (RE'12). It represents the latest in the long relationship between the RE conference series and the REJ, the primary conference series and scientific journal for reporting research in requirements engineering, respectively.

The requirements engineering research community is a genuinely international one, and in 2012, it was North America's turn in the RE conference's three-year cycle to host it. We chose Chicago and gathered in the University of Chicago's Gleacher Center during the 24-28 September. Chicago was an apt location as it has been the location of incredibly important advances in many fields ranging from physics to economics to architecture, and is a hugely significant financial, industrial, agricultural, journalistic and cultural center. More parochially perhaps, researchers at DePaul University, located within Chicago's loop, have made major contributions to RE research in recent years, so Chicago was an easy choice when we were looking for a location.

RE'12 attracted 137 paper submissions, and the four published here are extended versions of the best of the 106 that were submitted to the research track. RE submissions undergo a rigorous, two-stage review process, and although they were invited submissions, the extended papers were each required to undergo a further, independent review process before acceptance by the REJ.

P. Sawyer $(\square)$

Lancaster University, Lancaster, UK

e-mail: p.sawyer@lancaster.ac.uk
Of the four papers, the fist two directly address the theme of RE'12; uncertainty handling. Both papers make use of goal modeling, but otherwise take contrasting approaches.

Managing Requirements Uncertainty with Partial Models by Rick Salay, Marsha Chechik, Jennifer Horkoff and Alessio Di Sandro presents an approach for managing uncertainty based on partial requirements models. Here, uncertainty is represented explicitly as missing model elements and model annotations. Uncertainty is not, of course, static and the approach supports model refinement when uncertainties are resolved over time. While the approach is language independent, $i^{*}$ goal models are used to illustrate the use of annotations for uncertainty recording.

Assessing Requirements-Related Risks Through Probabilistic Goals and Obstacles by Antoine Cailliau and Axel van Lamsweerde presents a probabilistic approach for modeling risk in goal models. Probabilistic reasoning is the classical means for reasoning about uncertainty in all sorts of domains, but has been surprisingly little used in requirements engineering. This paper builds on the authors' established techniques for modeling obstacles to goal fulfillment, but augments them with a probabilistic layer that allows the risks associated with obstacles to be better understood and prioritized for treatment.

The two remaining papers focus on different, but equally live, problems.

A Cross-Domain Empirical Study and Legal Evaluation of the Requirements Water Marking Method by David Gordon and Travis Breaux addresses the tough problem of compliance with legal and regulatory requirements for a software system that sells into or is used in different jurisdictions. The authors' solution is called "requirements water marking," and the paper reports on two case studies used to empirically evaluate it. 
Features Meet Scenarios: Modeling and Consistency Checking Scenario-Based Product Line Specifications by Joel Greenyer, Amir Molzam Sharifloo, Maxime Cordy and Patrick Heymans lies within the intersection between requirements engineering and software product line research. This is a problematic region as requirements methods are generally poor at modeling and reasoning about variation and commonality. The authors' approach is to use a scenario-based technique for specifying product line requirements and a fully automated model-checking technique that allows inconsistencies to be identified.

I hope you will agree that these four papers not only represent exemplary research in requirements engineering, but also present results that advance the maturity of our discipline and the crucial role it plays in the modern world.

This special issue represents the final act of RE'12. It should not be forgotten that the paper presentation program from which these four papers derive is only one part of such an event. There are also workshops, tutorials, doctoral consortia, and a host of other events and happenings. I am indebted to many people for making RE'12 happen, including our three excellent keynote speakers; Chris Rupp, Nancy Leveson and Steve Fickas. Most important of all, however, were Jane Cleland Huang who handled local arrangements and our General Chair, Mats Heimdahl. 